\title{
JULIUS - An Extendable Software Framework for Surgical Planning and Image-Guided Navigation
}

\author{
Erwin Keeve ${ }^{1}$, Thomas Jansen ${ }^{1}$, Zdzislaw Krol ${ }^{1}$, Lutz Ritter ${ }^{1}$, \\ Bartosz von Rymon-Lipinski ${ }^{1}$, Robert Sader $^{2}$, Hans-Florian Zeilhofer $^{2}$ and \\ Peter Zerfass ${ }^{1}$ \\ ${ }^{1}$ Surgical Simulation and Navigation Group \\ research center $c$ a $e s$ a $r$ \\ Friedensplatz 16, 53111 Bonn, Germany \\ keeve@caesar.de \\ http: //www.caesar.de/ssn \\ ${ }^{2}$ Dept. of Cranio- and Maxillofacial Surgery \\ Technical University of Munich, Germany
}

\begin{abstract}
In this paper we introduce the extendable and cross-platform software framework JULIUS, which will become public available by the end of this year. JULIUS consists of three conceptual layers and provides diverse assistance for medical visualization, surgical planning and image-guided navigation. The system features a modular and portable design and combines both pre-operative planning and intra-operative assistance within one single environment.
\end{abstract}

\section{Introduction}

In image-guided surgery the vision of reality is enhanced using information from CT, MR and other medical imaging data. Certain instruments can be guided by these patient specific images if the patient's position on the operating table is aligned to this data $[1,2,3,4]$. In order to bring the actual computer-aided pre-operative planning scheme into the OR, it is best to use one single software environment for both the preoperative planning process and the intra-operative intervention. Since image-guided surgery is a relatively new field, advanced algorithms and techniques should easily be integrated into this environment. To reach this goal, we developed the extendable and cross-platform software framework JULIUS, which can be used as a general software framework for rapid application development as well as a front-end for image-guided surgery.

\section{Methods}

We developed JULIUS on top of the OpenGL graphics library using the Visualization Toolkit vtk for all visualization tasks and the Qt library for the user interface. JULIUS consists of three conceptual layers - see Figure 1: The Julius Software Development Kit (JSDK) builds the core application and controls the data processing pipeline. The Julius Graphical User Interface (JGUI) acts like a front-end to the JSDK and offers easy handling combined with time-saving functionalities to increase performance and

W. Niessen and M. Viergever (Eds.): MICCAI 2001, LNCS 2208, pp. 1336-1337, 2001.

(C) Springer-Verlag Berlin Heidelberg 2001 
productivity. The Julius Inter-Module Communication (JIMC) provides information exchange schemes and communication interfaces.

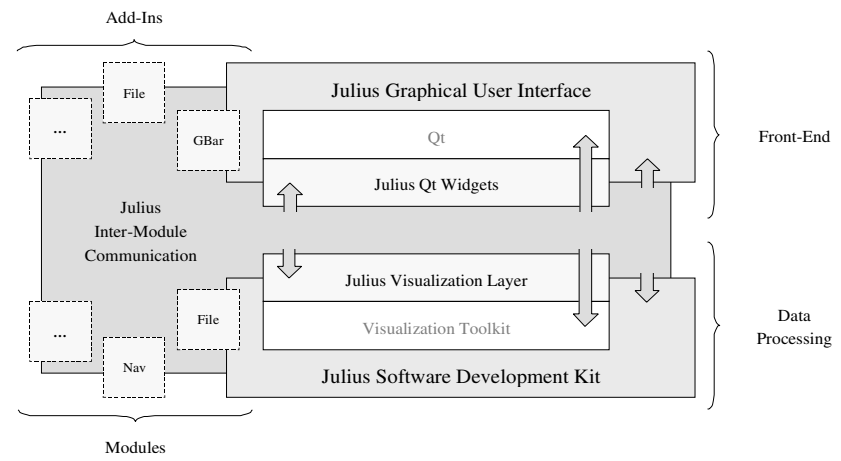

Fig. 1. The three conceptual layers of JULIUS, and the overall system architecture. Future developments can easily be integrated as modules or add-ins.

Fig. 2. The JULIUS graphical user interface: a.) The Global Bar window b.) The main window with $3 \mathrm{D}$ volume and/or surface visualization c.) The corresponding slice views

\section{Results and Conclusions}

With JULIUS we have created one single software environment for both pre-operative

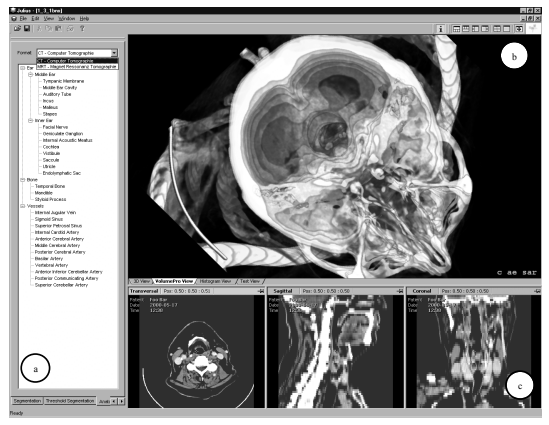
planning and intra-operative navigation achieving best results in routine surgical cases as well as in new computer-aided intervention methods. Modules for semi-automatic segmentation and registration, 3D reconstruction and visualization have been implemented. Interfaces to external devices like navigation and haptic systems are provided. The software framework has been tested in first surgical trials. We found that the separation between JSDK and JGUI enables on the one hand rapid software development for the scientific developer and on the other hand an intuitive handling for the surgeon. Application areas range from cranio- and maxillofacial surgery, to neurosurgery and orthopaedics. The clinical evaluation of the system in these areas will start within the next months.

\section{References}

1. ANALYZE, http://www.mayo.edu/bir/Analyze_Pages/Analyze_Main_TOC.htm

2. Gering D.T., "A System for Surgical Planning and Guidance using Image Fusion and Interventional MR," Master's Thesis, Massachusetts Institute of Technology, 1999.

3. Golland P., Kikinis R., Umans C., Halle M., Shenton M.E., Richolt J.A., "AnatomyBrowser: A Framework for Integration of Medical Information," In Proc. First International Conference on Medical Image Computing and Computer-Assisted Intervention; MICCAI'98, Cambridge, MA, pp. 720-731, 1998.

4. Leventon M.E., "A Registration, Tracking, and Visualization System for Image-Guided Surgery," Master's Thesis, Massachusetts Institute of Technology, 1997. 